ist, uber die Grenzen seines Faches hinauszugehen und aus der Beschäftigung mit den Resultaten der allgemeinen naturwissenschaftlichen Forschung täglich neue Anregungen zu entnehmen.

\section{Über Blaualgen.}

\author{
Ton Privatdozent Dr. Ernst G. Pringsheim, \\ Halle a. $S$.
}

Die Cyanophyceen oder Blaualgen stellen eine Gruppe niedrig stehender Organismen dar, deren mangelhafte physiologische Durchforschung sie aus bestimmten Gründen zum Gegenstand der verschiedensten Vermutungen gemacht hat. Thr einfacher Bau erinnert, wie das Ferdinand Cohn besonders hervorgehoben hat, in vielem an den der Bakterien. Man fabt deshalb auch die Spaltalgen (Cyanophyceen) und Spaltpilze (Bakterien) als Spaltpflanzen (Schizophyten) zusammen, Bezeichnungen; die auf die Art der Vermehrung durch Zellspaltung hinweisen. Was die Gestalt der Blaualgen anbelangt, so treten hier allerdings die einfachen Stäbchen und Kügelchen zurück gegen die Masse der Arten, die $z u$ einfachen Zellverbänden angeordnet sind, während unter den Bakterien die einfachsten Formen die Hauptmenge ausmachen. Aber das mag mit den Ernährungsverhältnissen zusammenhängen, die bei den Bakterien sich den echten Pilzen anschließen, während die Blanalgen Kohlensäure assimilieren wie die höheren Algen.

Nimmt man die Bakterien im weitesten Sinne als Einheit, so finden sich bei ihnen Parallelformen zu einer ganzen Anzahl von Cyanophyceengruppen. Cnd zwar sind es gerade die morphologiseh oder physiologisch abweichenden und eigenartigen Formen unter don Bakterien, die vielfach an Blaualgen erinnern. Nicht nur, daß die stickstoffsammelnde, merkwürdige Gattung Azotobakter blaualgenartig aussicht, auch unter den sogen. Purpurbakterien, die durch ibren Farbstoffgehalt und ihre ungeklärten Beziehungen zum Tichte unter den Bakterien eine Sonderstellung einnehmen, gibt es Arten. die gewissen Cyanophyceen gleichen. Besonders auffallend ist aber die Parallelität zwischen den blaugrünen Oscillarien und den Beggiatoren, die $z u$ den Schwefelbakterien gehören, d. h. zu jenen Organismen, die die Fähigkeit haben, Schwefelwasserstoff zu Schwefel und schließlich zu Schwefelsäure zu oxydieren und aus dieser Verbrennung ihren Bedarf an Lebensenergie zu bestreiten. Beide Gruppen sind in der Anordnung der Zellen, Gestalt und Bewegungsart völlig gleich. ohne sonst in der Organismenwelt ihresgleichen $\mathrm{zu}$ finden. Thre Zellfäden kriechen nämlich auf völlig rätselhafte Weise auf festen Unterlagen entlang, wobei nichts von Beweguagsorganen $z u$ erkennen ist. - Weitere Parallelformen zu nennen, erübrigt sich wohl.

SchlieBlich kommt noch den Bakterien und Blaualgen ein negatives Kennzeichen gemeinsam zu, nämlich der Mangel eines Zellkernes, der doch sonst bei allen tierischon wie pflanzlichen Zellen gefundon werden konnte. $Z$ war hat man neuerdings in den verschiedensten Strukturen in Bakterienzellen Kerne erkennen wollen, doch wurde diese Deutung höchstens für gewisse bei der Sporenbildung auftretende Körnchen wahrscheinlich gemacht, während bei Blaualgen und den manchmal sehr großen Zellen der Beggiatoren alle Bemühungen vergeblich waren. Es deutet dieser Mangel auf die allen Spaltpflanzen gemeinsame niedere Organisationsstufe hin, auf der offenbar die Arbeitsteilung in der Zelle noch nicht so weit fortgeschritten ist, wie bei den höher stehenden Organismen.

Neben diesen Ahnlichkeiten finden wir auch wichtige Organisationsmerkmale, die Spaltalgen und Spaltpilze unterseheiden. So geht den Cyanophyceen die Fähigkeit, innere Sporen zu bilden, und die, mit Hilfe ron ,Geißeln" frei im Wasser umher zu schwimmen, ganz und gar ab. Auch hat man ein gewisses Recht zu sagen, daß die charakteristischsten Parallelformen $z_{12}$ den Blaualgen unter den Spaltpilzen keine echten Bakterien seien. Trotzden hat wohl doch die Analogie mit deu Bakterien, die bekanntlich ,alles können“, dazu beigetragen, den Blaualgen allerlei Fähigkeiten zuzuschreiben, die bei der gavz ungenigenden physiologischen Durehforschung dieses Organismenstammes mehr Glauben genossen, als gut ist.

Noch ist die Erforsehung dieser Dinge erst in den Anfangsstadien, aber schon hat sich gezeigt, daB manches, was in den Büchern steht, einer strengeren Prüfung nieht stanthält. Man hat vermutet, daß Blaualgen darauf angewiesen seien, organische Stoffe zu verarbeiten und hat ihnen auf Grund unzureichender Beobarhtungen auch das Vermögen zugeschrieben, den Stickstoff der Luft zu verarbeiten. Schlieflich hat man auch geglaubt, annehmen zu dürfen, dab sie ihre Färbung je nach der Beleuchtung andern, so dab sie immer die Strahlen absorbieren und zur Assimilationsarbeit heran" ziehen sollen, die ihnen an einem bostimmten Orte geboten werden. Diese drei Punkte sollen auf Grund neuer Untersuchungen hier besprochen werden.

Zunächst sei noch betont, dab die genannten physiologischen Fragen bei der Manuigfaltigkeit der ökologischen Bedürfnisse innerhalb des Cyanophyceenstammes dureh das Studium weniger Arton nicht mit Sicherheit algemein entschieden worden können. Wir keumen nänlich Blaualgen aus den reinsten Quellbächen einerseits, aus stark verschmutzten Wassern andererseits; ferner solche, clje in heißen Quellen, und solche, die als Planktonformen in kalten Meeren und Gebirgseen auftreten. Auch in Gesellschaft anderer Lebrwesen treten sie auf, als Flechtengonidien mit Pilzen zusammen, als Endophyten in Azolla, Guniera, Icbermoosen und Cykadeen. Aus dieser Aufzählung dïrfte schon hervorgchen, auf welche Manniefaltigkeit auch der physiologisehen Eigensehaften gerechnet werden muB. Diese zu erforschen, gelang aber bisher deshalb nicht, weil man die Blaualgen nicht von anderen, mit ihnen zusammenlebenden Organismen, vor allem Bakterien, zu trennen vervochte; Stoffwechsel- und Emährungsversuche 
sind natürlich stets nur an einheitlichem Materiale mit Erfolg anzustellen.

Es ist mir nun gelungen, drei Arten von Cranophyceen mit Kriechvermögen, nämlich zwei Oscillarien und ein Nostoc in absoluter Reinkultur, $d$. h. in Abwesenheit aller fremden Organismen, auch der Bakterien, zu züchten ${ }^{1}$ ).

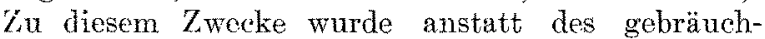
lichen Agar-Agars eine Schicht von kolloidaler Kieselsäure mit den nötigen Nährsalzen verwendet, Auf dieser breiteten sich die Blaualgen aus und streiften sich beim Gleiten über die Gallertoberfläche die größte Masse der anhaftenden Bakterien ab. Letztere fanden darauf auch keine Nährstoffe, während sich die Algen am Tichte gut vermehrten. So konnte schließlich an Stellen, die vom Orte der ursprünglichen Impfung entfernt lagen, ein schon mit weniger Bakterien behaftetes Material entnommen und durch Wiederholung der gleichen Behandlung weiter gereinigt werden. Ganz zu beseitigen rermochte man die Bakterien auch so nicht, doch befreiten sich wenigstens einzelne Fäden schlieflich ron ihnen. Sie konnten durch tibertragen auf Agar mit organischen Stoffen herausgefunden und isoliert werden, womit die Reinkultur erzielt war.

Mit dem so gewonnenen und leicht zu vermehrenden Material wurden nun systematische Ernährungsversuche angestellt, die ein leidlich einheitliches Bild ergaben. In rein ,mineralischen" Nährlösungen ohne organische Stoffe gediehen die kultivierten Arten bei neutraler oder schwach basischer Reaktion mit Nitraten wie mit Nitriten und Ammonsalzen als Stickstoffquellen. Ebenso konnten dieverschiedenartigsten organischen Stickstoffverbindungen, wie z. B. Eiweilstoffe und deren Abbauprodukte ausgenutzt werden, ohne aber gegenüber den anorgamischen irgendwelche Vorzüge aufzuweisen. Von anderen Kohlenstoffrerbindungen wurden organische Säuren, höhere Alkohole, Kohlehvdrate usw. geprïft. Alle organischen Stoffe erwiesen sich schon bei recht niederer Konzentration als schädlich, bei noch geringerer aber als nahezu unwirksam. Nur selten, so z. B. bei manchen Zuckerarten, komnte eine schwache Förderung gegenïber mineralischen Lösungen festgestellt werden. Im ganzen ist also eine erhebliche Bedeutung organischer Stoffe für die untersuchten Arten nicht anzunehmen. So kann es auch nieht wundernehmen, daß eine Ernährung nur mit solehen, also unter Ausschlub der Kohlensäureassimilation bei Dunkelkultur nicht gelungen ist. Eine Vermehrung im Finstern ist für eine endophytische Art behauptet worden. Da diese unter natïnlichen Umständen in sehr schwacher Beleuchtung gedeiht and von der Wirtsyflanze vielleicht organische Nahrung bezieht, muß die Möglichkeit zugegeben werden.

Was das Stickstoffbindungsvermögen der Blaualgen anbelangt, so sind dahingehende Ver-

1) E. G. Prinfsheim, Kulturversuche mit chlorophyllfültrenden Mikroorganismen, ITI. Mitteilung: Zur Physiologie der Schizophyceen. Cobns. Beiträge z. Biol. d. Pflanzen, Bd. $X I I, 1013$, S. 49. mutungen hauptsächlich von Beijerincki1) ausgesprochen worden. Er fand nämlich, daß in Leitungswasser mit einer Spur Gartenerde sich eine ïppige Cyanophyceenvegetation entwickcln kann, obgleich die vorhandenen Mengen an Stickstoffverbindungen sehr gering sind. Neue Versuche von. Glade? zeigten aber, daB die in solchen Kulturen auftretenden Arten sich nicht mehr zu vermehren vermögen, wenn sorgfältig gereinigte Salze und destilliertes Wasser verwendet werden. Eine minimale Menge von Stickstoffsalzen dagegen erlaubt reichliche Entwiellung. Die meisten Blaualgen brauchen hingegen höhere Stickstoffmengen zum Gedeihen, wenn sie auch immer noch recht bescheiden sind. Wiederum ist die Frage nicht für alle Arten als entschieden zu betrachten, wenn auch dic Wahrscheinlichkeit nicht sehr grob ist, dab Blaualgen erheblichere Mengen von Luftstickstoff festlegen, da gerade die von Beijerinch genannten nicht dazu imstande sind. Sie dürften vielmehr häufig mit stickstoffbindenden Bakterien vergesellschaftet sein.

SohlieBlich zu der Erscheinung der sogenannteu komplementären chromatischen Adaptation von Engelmann und Gaidukow. Nach einer geistreichen Hypothese des crsteren haben die das Chlorophyll bei vielen Algen begleitenden Farbstoffe, die die rote Farbe der Rhodophycen, dio braune der Phaeophyceen usf. bedingen, die Aufgabe, sonst nicht ausnutzbare Lichtstrahlen durch Absorption der Kohlensäureassimilation dienstbar zu machen. Vor allem soll das für die kurzwelligen Strahlen gelten, die ins Meerwasser tiefer eindringen als die langwelligen und von den gelben, braunen und roten Farbstoffen der Algen stärker absorbiert werden als yom bloßen Ohlorophyll.

Eine starke Stutze dieser Hypothese schien es nun $z u$ sein, als sich zeigte, daB die nicht immer blaugrünen, sondem vielfach alle möglichen Farben zeigendeu Spaltalgen unter wechselnden Umständen ihre Farbe zu verändern imstande sind. Der Farbenwechsel wird durch das Zugegensein eines blauen uad eines gelben bis roten Farbstoffes neben dem Chlorophyll ermöglicht, deren Mischungsverhältnis von Fall zu Fall variiert. Gaiduloow glaubte nun. durch Änderung der Farbe des die A]gen treffenden Lichtes eine gosetzmäßige Umfärbung hervorrufen zu können, und zwar in dex Weise, daß die erzielte Farbe der Spaltalgen an nähernd komplementär zu der der Beleuchtung würde. Es würde dam mehr von dem farbigen Lichte absorbiert als bei normaler Färbung, was für die Pflanzen zweckmäBig wäre und die für die genannten Meeresalgen aufgestellte Hypothese stützen wiirde.

Leider konnten die Gaidukowschen Versuche in zwei umabhängig voneinander angestellten Untersuchungen nicht mit Frfolg wiederholt werden.

1) I. W. Beijerinels, tber oligonitrophile Bakterien, Centralblatt fïr Bakt., II. Abt., Bd. VII, 1901, S. 561.

2) Noch nicht veroffentlicht.

3) N. Gaidukou, Die Farbenänderung bei den Prozessen der komplementäen chromatischen Adaptation. Ber. d. deutsch. bot. Ges., Bd. XXI, 1903, S. 519. 
Weder Magnus und Schindlert) noch ich selbst bekamen eine komplementäre Umfärbung. Vielmehr zeigte sich, daß die Farbe der Blaualgen bei Stickstoffmangel und in $z u$ starker Beleuchtung ausbleicht und sich mehr dem Gelb nähert. Unter günstigen Umständen tritt das fast oder ganz verschwundene Blattgrün sowie der blaue Farbstoff wieder auf, was aber sogar im Dunkeln vor sich gehen kann, wie von Boresch') gezeigt wurde. Mit der Farbe der Beleuchtung hat also die Verfärbung offenbar nichts zu tun. Sie reiht sich vielmehr dem Vergilben vieler anderer Algen und grüner Flagellaten unter den gleichen Umständen ein, das schon längere Zeit unter der Bezeichnung des Etiolements aus Stickstoffhunger bekannt ist.

Wir sehen also, daß die Cyanophyceen nicht alle die merkwürdigen Fähigkeiten besitzen, die man ihnen zugeschrieben hat, und sich von anderen Algen in ihren physiologischen Eigenschaften wenig unterscheiden. Nur ihre Anspruchslosigkeit und Widerstandsfühigkeit gegen extreme Einflüsse befähigen sie zu der so verschiedenartigen Lebensweise. $\mathrm{Da}$ aber bisher nur wenige Arten genaver untersucht werden konnten, so sind zukünftige tberraschungen nicht ausgeschlossen. Auch dürfte eine Erforschung der speziellen Bedürfnisse und Anpassungsbreiten bei Blaualgen der Standortslehre und Okologie sehr zugute kommen.

\section{Die Radioaktivität der Heilquellen.}

Von Professor Dr. H. Sieveling, Karlsruhe.

Die heilkräftige Wirkung der Thermalquellen, die sich seit alten Zeiten eines besonderen Rufes erfreuten, ist durch die Entdeckung des Radiums in ein ganz neues Licht gerückt worden. Seit den Tagen der Römer, die bekanntlich in der Bädertechnik unübertroffen dastehen, haben Tausende Heilung und Linderung ihrer Gebrechen in den stärkenden Quellen gesucht. Der Ruf der Wunderkraft erbte sich durch Geschlechter hindurch fort. Die Erklärung hat im Laufe der Zeiten manche Wandlung erfahren. Wo nicht ein ausgeprägter Salzgehalt zutage trat, wo ferner keine hohe Temperatur die Quelle vor anderen auszeichnete, suchte man den ,Brunnengeist" durch Spuren von Gold oder anderen wertvollen Beimengungen $\mathrm{zu}$ erklären. Poetischer, wenn auch nicht naturwissenschaftlich, war die Erklärung durch ein sagenhaftes Ereignis, dem die Veredelung der Quelle zu danken sein sollte. Schon die Schriftsteller des Mittelalters lassen es als wahrscheinlich gelten, dal irgend etwas Verborgenes in den Quellen liege, da nur an Ort und Stelle die volle Wirkung zur

1) W. Magmus u. B. Schindler, Über den EinfluB der Nährsalze auf die Färbung der Oscillarien. Ber. d deutsch. bot. Gesellsch., Bd. $\bar{X} X \bar{X}, 1912$, S. 314.

$\left.{ }^{2}\right) K$. Boresch, Die Fürbung von Cyanophyceen und Chlorophyceen in ihrer Abbängigkeit vom Stickstoffgehalt des Substrates. Jahrb. f. wissensch. Botanik, Bd. $L I I, 1912$, S. 145 .
Geltung komme. In neuerer Zeit hat Heinrich Heine treffend die Heilquelle mit einem Volkslied verglichen. Will der Chemiker im Laboratorium das Wasser künstlich nachahmen, so glückt ihm das ebensowenig, wie einem neueren Dichter die Nachahmung eines Volksliedes. Es fehlt das hypothetische Fluidum. Bekannter mag die Anekdote von Liebig sein, der nur mit großem Mißtrauen an eine Kur in Gastein heranging, da eine Vorprüung des Wassers ihm die totale Neutralität verraten hatte. Destilliertes Wasser könne er zu Hause billiger haben, meinte er nicht ganz mit Uarecht. Erst nach erfolgreicher $\mathrm{Kur}$ lieB ex sich bekehren und auberte, es müsse etwas Elektrisches oder Magnetisches in der Quelle sein. Und damit hat er anscheinend teilweise das Rechte prophetisch erkannt. Er ahnte noch nichts vom Radium; hätte er aber in Gastein ein Elektroskop gehabt, so wäre ihm sicher nicht verborgen geblieben, daß Luft, die mit diesem Wasser in Berührung gewesen, ein hohes elektrisches Leitvermögen besitzt.

Gleichzeitig und unabhängig voneinander haben Allen und Himstedt darauf hingewiesen, daß die Heilwirkung auf den Gehalt an Radiumemanation zurückzufüren sei. Wie ungezwungen erklärt sich von diesem neuen Standpunkt aus das Versagen der Heilkraft fern vom Ort der Quellenmündung. Gäbe es ein Maß dafür, so hätte man eine Abnahme der Heilwirkung mit einer Halbwertszeit von 3,85 Tagę konstatieren können! Und ebenso die mißglückten Versuche, das Heilwasser künstlich nachzuahmen; es fehlte eben die radioaktive Emanation.

Bekanntlich ist diese in der Natur ungemein verbreitet. Die Leitfähigkeit dex Luft, ohne welche die erdelektrischen Vorgänge gar nicht zu erklären sind, beruht zum großen Teil auf der Gegenwart der Emanation. Der Boden atmet dieselbe ein und aus, entsprechend den Schwankungen des Luftdruckes. Auf dem Festlande wie auf dem Ozean, am Boden wie in einigen Kilometer Höhe auf Bergen oder im Ballon, überall findet sich das radioaktive Gas. Wir sind sogar berechtigt zu der Annahme, dab die Erdwärme ihre Verluste aus der Energie des Radiums deckt.

Fast jede Quelle zeigt bei sorgfältiger Prüfung einen Gehalt an Emanation. Eine Quelle ohne jede Spur von Radium ist eine Seltenheit, gewissermaßen ein Naturwunder. Bei anderen Quellen wieder sind die mitgeführten Beträge recht beträchtlich. Leider ist es in den meisten Fällen nur Emanation, die in kurzer Zeit sich umsetzt. Die Salze des Radiums sind meist sehr schwer löslich, und nur minimale homöopathische Spuren finden sich gelegentlich im Wasser. Eine erfolgreiche Ausbeutung der Radiumschätze der Erde auf diesem Wege ist leider ein Ding der Unmöglichkeit.

Neben der Emanation des Radiums findet sich häufig auch die des Thoriums. So ist der Charakter jeder Quelle ein ganz bestimmter, und das mag bei der Heilwirkung eine große Rolle spielen. Die Natur bleibt hier wieder die unübertroffene Meisterin. 